\title{
Chaos, Adaptation and Information are the Main Pillars of Carcinogenesis
}

\author{
Mesut Tez M.D ${ }^{1, *}$ and Selda Tez M.D ${ }^{2}$ \\ 1 Ankara Numune Hospital Department of Surgery, Samanpazarı 06100 Ankara/TURKEY \\ 219 mayis Hospital,Department of Radiology, Dikmen, Ankara/TURKEY /TURKEY \\ * Correspondence: mesuttez@yahoo.com; Telephone: +90(532)416-06-69 \\ Address: Ankara Numune Hospital Department of Surgery, Samanpazarı 06100 Ankara/TURKEY
}

\begin{abstract}
Cancer, a disease of multicellular organisms, probably developed almost immediately following the transition from unicellular to metazoan life, about one billion years ago. Great efforts have been made to understand the carcinogenesis for many years. In this paper, We tried to explain the cancer based on "chaos", "adaptation" and "information" with the context of new literature findings.
\end{abstract}

Keywords: cancer; adaptation; chaos

\section{Introduction}

Despite the spectacular contributions to knowledge made by molecular biology during the last half century small variations in cancer mortality was observed [1,2]. There are a number of theories of carcinogenesis and these theories may be used to justify various alternative cancer treatments. Somatic mutation theory (SMT) is the most popular theory [3]. Mutational changes generally would be insufficient to cause cancer, because a minority of cancers were only triggered by about $5 \%$ mutations [4]. Another alternative theory is Tissue Field Organization Theory (TOFT). According to TOFT, the main mechanism under the carcinogenesis is corrupted tissue organization. Carcinogens destroy the normal tissue structure thus disrupting cell-to- cell signaling and genomic integrity [3]. Hence, in TOFT the DNA mutations are the effect, not the cause of the tissue-level events [5,6]. These two theories do not contradict each other but they converge and complement each other in a single unified carcinogenesis theory [6]. We proposed a new hypothesis: that cancer arise from the adaptation of the stem cell (chaotic adaptation theory), 10 years ago [7-9]. Main pillars of the theory are "chaos", "adaptation" and "information".

\section{Background}

\section{Homeostasis}

Normal epithelium of the tissues has a turnover. Life span of the epithelial differentiated cell is very short. For example, lifespan of colonic epithelium cell is 3-4 days. Even within this short period, the differentiated epithelial cells are constantly in contact with the stem cells Normal somatic stem cells (SSCs) are described as immature cells that have the double capability of self-renewal and differentiation potential. Somatic stem cells generate differentiated progenies at regular basin. Somatic stem cells are maintained throughout life. Somatic stem cells-progeny turnover controlled by feedback loops [10,11]. 
division and the other reduces the probability of SSCs self-renewal, leading to cell death via terminal differentiation. $[10,12]$. Somatic stem cells are thought to often represent the targets for cancer initiation. Somatic stem cells in tissues also appear to be positioned in protective locations (niche). For example, blood stem cells are localized in the bone marrow, and gut stem cells are positioned distant from the nasty contents of the large intestine which should reduce exposure to carcinogens. Niche has usually been defined as the location surrounding the stem cells. However, this definition of niche is being changed to involve the cellular components, such as fibroblasts, endothelial cells and immune cells, which are rich in extracellular matrices, and secreting factors and receptors as well as the signs of the microenvironment affected by the metabolism [13].

Healthy SSCs should serve to maintain the status quo [14]. Briefly, SSCs activity may be accounted for by natural selection acting on the decisions of SSCs in response to the signals from other SSCs in the local micro-environment, and from the more differentiated cells in the rest of the organism [15]. On the other hand, SSCs show a remarkable regulatory flexibility that allows them to thrive under different (previously unknown) external conditions and to survive harsh situations. This ability to constantly sense and adapt to environmental changes is important for all organisms to maintain cellular functions [16]. Local, systemic and environmental factors regulating of the SSCs [17].

The behavior (phenotype) of SSCs is controlled by coordinated activation and/or inhibition of thousands of genes. This coordination is achieved via a complex network of gene regulation that enables a cell to express the appropriate set of genes for a particular environment and/or phenotype [18]. The genome just needs to have enough instructions to recreate the required microenvironment so that the behavior can be sufficiently replicated within the required space to serve the required function. Signaltransduction pathways can translate extracellular signals into specific intracellular responses, including the launch of alternative gene expression programs to cope with new conditions. However, in a nonlinear system, it is not necessary for the genome to record every detailed instruction; in fact, the genome lacks the necessary power to encode everything wide range of challenges (stress), including those not encountered during evolutionary history [19].

Stress

Stress (microenviromental stress or field cancerization) is defined as an environmental factor that causes a change in a biological system which is potentially injurious [20]. Homo sapiens currently encounter a plethora of new chemical and physical agents (mutagen or non mutagen) that were not present in nature throughout our long evolutionary history. [21]. We are currently exposed to such as vinyl chloride, dioxins or benzene (all shown to cause cancers) are also exposed to amounts of ionizing radiation, which far exceed natural levels experienced by our ancestors. Our enzymatic detoxification systems can still recognize functional groups within these molecules; they are not completely efficient in guarding our bodies from the onslaught of foreign molecular insults [21]. At the same time, we are getting our calories mostly from sugar and high-fructose corn syrup and getting much less exercise [22,23]. Stress shortens the lifespan of differentiated cell and SSCs rapidly and reversibly switch to produce an excess of progeny to regenerate the lost tissue [24].

\section{Chaotic behavior}

Chaotic behavior is highly widespread in the universe such as in weather, road traffic, meteorology, anthropology, sociology, physics, computer science, economics and even biology [25,26].

From an evolutionary perspective, self-organization creates complex systems which are then shaped until they exist at the boundary between order and chaos, held there by an intricate system of feedback. The system can use deterministic chaos when needed and then return to order, maintaining its flexibility 
without losing its structure [19]. Several studies have demonstrated an important role of feedbacks in generation of chaos [27]. Changes in the chaotic dynamics of cellular function evolve in parallel with changes in the fractal geometry of cellular structure [19].

\section{The Hypothesis}

In physiologic conditions, somatic stem cells (SSCs) receive information from their progenies. This received information is processed by SSCs genome and appropriate response is created. This response is production of new progenies. There is no time delay for the creation of appropriate response and production of new progenies (feed-back). We named this phase as a physiologic phase [28]. In second stage, known extreme conditions (inflammation, heat, chemicals etc.) or previously unknown conditions caused to phenotypic diversity (Tactical phase). Atrophic and hypertrophic changes are seen in this phase. In this phase, SSCs create minimally different phenotypes. SSCs increase or decrease size of progenies and changes metabolic properties.

Genotypes can be organized into vast genotype spaces [29]. "A" new pathway/phenotype can mean the difference between life and death; either by allowing its carrier to subsist survives in a hostile environment (innovative ecosystem). If tactical phase is not enough to protect progenies, SSCs genome jumps to edge of chaos (Edge of chaos phase or atavistic phase).[30] Previously known differentiated cells (progenies) originated from the SSCs at this stage (transition of epithelium another type of resistant epithelium). In this state the system can be predicted and controlled. This phase represents as dysplasia or metaplasia (preneoplastic) clinically. These phenotypes may be evaluated as Atavistic phenotypes [31].

If the SSCs and progenies cannot adapt the hostile environment (increased free energy), systems jump to chaotic phase or rescue phase (innovative phase). In this phase, SSCs become cancer stem cells. The lag in transduction of the input signal is a necessary condition for emerge of chaotic behavior $[32,33]$ These changes are "Strategic" changes (fundamental changes in ongoing cell function). SSCs explore own genotype spaces through "chaotic search".

Stochasticity and chaos are not identical. Chaotic sequences can be produced by determinate algorithms as first shown by Lorenz. The difference is important because the variations in determinate chaos are constrained by an attractor, whereas genuine stochasticity is not. The difference can be made clear in phase plots. This dependence of initial conditions may explain tissue specific cancer behavior (e.g. different behavior of colorectal cancer vs. pancreas cancer).

All chaotic innovations originate in some space of genotypes. These innovations involve molecules with new structures and biochemical functions. Chaos can act as a "heterogeneity engine" that allows a population of cells to quickly explore a large number of phenotypes (different morphology, nuclear structure, chromatin architecture, metabolism, trans-membrane potentials etc ) [28]. Genome chaos refers to elevated rates of genomic restructuring, such as changes in chromosome number (aneuploidy), segmental chromosomal rearrangements (translocations, duplications, inversions and deletions), instability of repetitive sequences [32], and single catastrophic events, such as the recently described phenomenon of chromothripsis [33].

In this stage; SSC's choices "survive" instead of "evolve". Indeed, molecular differences can usually be found between any two cases, leading to the impression that perhaps, like snowflakes, no two cancer cases are exactly alike. Lack of experimental evidence of chaos generation at the intracellular level in vivo may indicate that during evolution the cell has found a solution to this problem the possibility of stabilizing the system in the presence of chaos-generating factors [34]. Phenotypic diversity created by genome chaos can be advantageous in hostile environments is well established in the ecology and 
population genetics literature [35]. Thus, it seems that previously unknown stress can trigger chaotic, genome expression responses on the basis of regulatory network plasticity, followed by selection for adaptive portions of the response [30,36]. Also, chromosomal chaos silences immune surveillance [37]. Despite the fact that genome chaos is often associated with elevated levels of cell death some chaotic genomes temporarily survive. The phenotype of the malignant progenies appears to depend on both the tissue specific microenvironment and the organ specific genome space, as demonstrated by previous experiments [38].

Niche construction is the process by which organisms modify their environment through their normal bioregulatory activity. Tumor tissue is niche construction. Metastases can be explained by the adaptive walk requires that some members of a niche venture out to see what other niches have to offer. New niches might be more adaptive to their circumstances than the present niche, other new niches less so.

In presence of germline mutations or acquired mutations, there are wrong SSCs in right place (absent of the SSC receptors for environmental signals) and aforementioned mechanism starts again.

Approximately $45 \%$ of the human genome is comprised of transposable elements (TEs). TEs are not simply 'parasitic' DNA. TEs activity can play an essential role in the host response to stress, facilitating the adaptation of populations and species facing changing environments. TEs have been shaping the genome throughout evolution, contributing to the creation of new genes and sophisticated regulatory network systems [8]. TEs can also facilitate this chaotic genome reorganization.

In addition, the process of aging is characterized by functional decline due to histological and biochemical changes in tissues and organ systems with the passage of time. Declining functionality is paralleled by diminishing capacity to respond to injury or stress and this chaotic behavior may be the way of fight against stress for aged SSCs. This may explain age-dependent rise in cancer incidence.

\section{Evidence supporting the chaotic adaptation theory}

Liu et al. demonstrated that, potential chaotic motifs are seen in the expression of certain genes in the cancer cell line and they conclude that more complex and somewhat more disorganized chaotic motifs can be found in cancer [18]. Ghosh et al. considered a mathematic model, describing the interactions of cancer cells with their microenvironment and they demonstrated chaotic behavior of cancer cell [39]. Uthamacumaran published an interesting hypothetical article. In this article, author tried to explain cancer with quantum chaos concept and stated that "A multi-dimensional model of quantum evolution by adaptive selection has been established for cancer biology" [40]. Fractal entropy is a model recently proposed by Garland which again places metabolism centre stage in cancer development. Garland also pointed out the paradox, discussed above, regarding the common profile of cancer cells, and noted that many of the genetic and epigenetic pathways involved in cancer development either directly or indirectly also influence energy management [41].

Also several articles indicate the importance and significance of chaos/adaptation in the development and prognosis of cancer [42-44].

\section{Consequences of the hypothesis and conclusion}

According to CAT, the development of tumors is closely related to the microenvironment; therefore, the treatment and prevention of cancer can be achieved by changing the microenvironment. We think that development of drugs or agents fix the degenerated microenvironment will be use to prevent or treat the cancer $[45,46]$. 


\section{References}

1. Ducasse, H., et al., Cancer: an emergent property of disturbed resource-richenvironments? Ecology meets personalized medicine. Evolutionary applications, 2015. 8(6): p. 527-540.

2. Heng, H.H., Debating cancer: the paradox in cancer research. 2015: World Scientific.

3. Sonnenschein, C. and A.M. Soto. Theories of carcinogenesis: an emerging perspective. in Seminars in Cancer Biology. 2008. Elsevier.

4. Vaux, D.L., In defense of the somatic mutation theory of cancer. Bioessays, 2011. 33(5): p. 341343.

5. Sonnenschein, C. and A.M. Soto, Somatic mutation theory of carcinogenesis: why it should be dropped and replaced. Molecular Carcinogenesis, 2000. 29(4): p. 205-211.

6. Rosenfeld, S., Are the somatic mutation and tissue organization field theories of carcinogenesis incompatible? Cancer informatics, 2013. 12: p. 221.

7. Tez, M. and S. Tez, Is cancer an adaptation mechanism to stress? Cell Biology International, 2008. 32(6): p. 713.

8. Tez, S. and M. Tez. Cancer is The Chaotic Search For Adaptation To Previously Unknown Environments. in Theoretical Biology Forum. 2016. Fabrizio Serra.

9. Tez, M. Complex system perspective in colorectal carcinogenesis. in Theoretical biology forum. 2011.

10. Rodriguez-Brenes, I.A., N.L. Komarova, and D. Wodarz, Evolutionary dynamics of feedback escape and the development of stem-cell-driven cancers. Proceedings of the National Academy of Sciences, 2011. 108(47): p. 18983-18988.

11. Li, J.-W., et al., Tumors arise from the excessive repair of damaged stem cells. Medical Hypotheses, 2017. 102: p. 112-122.

12. Tata, P.R. and J. Rajagopal, Regulatory Circuits and Bi-directional Signaling between Stem Cells and Their Progeny. Cell Stem Cell, 2016. 19(6): p. 686-689.

13. Scadden, D.T., Nice neighborhood: emerging concepts of the stem cell niche. Cell, 2014. 157(1): p. 41-50.

14. Casás-Selves, M. and J. DeGregori, How cancer shapes evolution and how evolution shapes cancer. Evolution: Education and outreach, 2011. 4(4): p. 624.

15. Mangel, M. and M.B. Bonsall, Phenotypic evolutionary models in stem cell biology: replacement, quiescence, and variability. PLoS One, 2008. 3(2): p. e1591.

16. Braun, E., The unforeseen challenge: from genotype-to-phenotype in cell populations. Reports on Progress in Physics, 2015. 78(3): p. 036602.

17. López-Lázaro, M., Understanding cancer: 15 questions and answers. ResearchGate, 2016.

18. Liu, D., L. Albergante, and T. Newman, Universal attenuators and their interactions with feedback loops in gene regulatory networks. bioRxiv, 2016: p. 074716.

19. Sharma, V., The Application of Chaos Theory and Fractal Mathematics to the Study of Cancer Evolution: Placing Metabolism and Immunity Centre Stage. Medical Research Archives, 2016. 4(6).

20. Hoffmann, A.A. and P.A. Parsons, Evolutionary genetics and environmental stress. 1991: Oxford University Press.

21. Smith, A.J., J. Oertle, and D. Prato, Environmental Carcinogens and the Kinds of Cancers They Cause. Open Journal of Oncology, 2014. 3(1).

22. Ruiz-Núñez, B., et al., Lifestyle and nutritional imbalances associated with Western diseases: causes and consequences of chronic systemic low-grade inflammation in an evolutionary context. The Journal of nutritional biochemistry, 2013. 24(7): p. 1183-1201.

23. Langie, S.A., et al., Causes of genome instability: the effect of low dose chemical exposures in modern society. Carcinogenesis, 2015. 36(Suppl 1): p. S61-S88.

24. Wabik, A. and P.H. Jones, Switching roles: the functional plasticity of adult tissue stem cells. The EMBO Journal, 2015: p. e201490386.

25. Rao, B., et al., How do biological systems escape 'chaotic'state? Journal of Biosciences: p. 1- 2.

26. Scharf, Y., A chaotic outlook on biological systems. Chaos, Solitons \& Fractals, 2017. 95: p. $42-47$. 
27. Likhoshvai, V.A., et al., On the chaos in gene networks. Journal of Bioinformatics and Computational Biology, 2013. 11(01): p. 1340009.

28. Suzuki, Y., et al., Periodic, quasi-periodic and chaotic dynamics in simple gene elements with time delays. Scientific reports, 2016. 6.

29. Wagner, A., The origins of evolutionary innovations: a theory of transformative change in living systems. 2011: OUP Oxford.

30. López-Maury, L., S. Marguerat, and J. Bähler, Tuning gene expression to changing environments: from rapid responses to evolutionary adaptation. Nature Reviews Genetics, 2008. 9(8): p. 583.

31. Davies, P.C. and C.H. Lineweaver, Cancer tumors as Metazoa 1.0: tapping genes of ancient ancestors. Physical biology, 2011. 8(1): p. 015001.

32. Agur, Z., et al., Disruption of a Quorum Sensing mechanism triggers tumorigenesis: a simple discrete model corroborated by experiments in mammary cancer stem cells. Biology Direct, 2010. 5(1): p. 20.

33. Weiss, H., A Mathematical Introduction to Population Dynamics. 2009: IMPA.

34. Khlebodarova, T.M., et al., Chaos and hyperchaos in simple gene network with negative feedback and time delays. Journal of Bioinformatics and Computational Biology, 2017. 15(02): p. 1650042.

35. Kussell, E. and S. Leibler, Phenotypic diversity, population growth, and information in fluctuating environments. Science, 2005. 309(5743): p. 2075-2078.

36. Ferriere, R. and G.A. Fox, Chaos and evolution. Trends in ecology \& evolution, 1995. 10(12): p. 480-485.

37. Zanetti, M., Chromosomal chaos silences immune surveillance. Science, 2017. 355(6322): p. 249250.

38. Afify, S.M., et al., A model of CSC converted from iPSC in the conditioned medium of HCC paving the way to establish HCC CSC. 2018, AACR.

39. Ghosh, D., et al., How tumor growth can be influenced by delayed interactions between cancer cells and the microenvironment? Biosystems, 2017. 158: p. 17-30.

40. Uthamacumaran, A., A biophysical approach to cancer dynamics: Quantum chaos and energy turbulence. Biosystems, 2017. 156: p. 1-22.

41. Garland, J., Energy management-a critical role in cancer induction? Critical Reviews in Oncology/Hematology, 2013. 88(1): p. 198-217.

42. Rangel, N., M. Forero-Castro, and M. Rondón-Lagos, New insights in the cytogenetic practice: Karyotypic chaos, non-Clonal chromosomal alterations and chromosomal instability in human cancer and therapy response. Genes, 2017. 8(6): p. 155.

43. Bi, P., S. Ruan, and X. Zhang, Periodic and chaotic oscillations in a tumor and immune system interaction model with three delays. Chaos: An Interdisciplinary Journal of Nonlinear Science, 2014. 24(2): p. 023101.

44. Tez, M., Is cancer an adaptation of the adult stem cell? Medical Hypotheses, 2006. 66(2): p. 448449.

45. Zhou, S., et al., Reprogramming Malignant Cancer Cells toward a Benign Phenotype following Exposure to Human Embryonic Stem Cell Microenvironment. PloS one, 2017. 12(1): p. e0169899.

46. Tarasewicz, E., et al., Embryonic Stem Cell Secreted Factors Decrease Invasiveness of TripleNegative Breast Cancer Cells Through Regulome Modulation. Cancer biology \& therapy, 2017(justaccepted): p. 00-00. 